gastric carcinoma. One of its major virulence determinants is cytotoxin associated gene $\mathrm{A}, \mathrm{cag} A$, and high levels of $\mathrm{cag} A$ expression are associated with more severe disease. The functional promoter elements of cag $A$ in the $436 \mathrm{bp} c a g A B$ intergenic region have been previously analysed but differences in the promoter region and levels of cagA expression between different $H$ pylori strains have not been studied in detail. We aimed to analyse the cagA promoter region to determine whether naturally occurring polymorphic differences within it contribute to differences in cagA expression level.

Methods Biopsy samples were obtained from 17 patients undergoing routine upper GI endoscopy at the Queen's Medical Centre Nottingham, UK. RNA was extracted directly from biopsies and cagA expression levels were analysed by real-time qPCR. The cagAB intergenic region of all 17 clinical strains were sequenced and aligned using the ClustalW multiple sequence alignment program and ranked in order of cagA transcript levels. A potentially relevant natural mutation was then created artificially by site-directed mutagenesis to prove its importance.

Results A potentially important polymorphism was identified within an imperfect inverted repeat where an A was commonly replaced by a $T$ at position -54 . Strains possessing $T$ at this position expressed higher cagA mRNA levels than those with an A ( $p=0.016)$. To test whether this was a direct determinant of cagA transcription level, a mutation was engineered at position -54 ( $\mathrm{T}$ to $\mathrm{A}$ ) in high transcription strain 83 . This resulted in a $30 \%$ reduction in cag $A$ transcript level when compared to an isogenic control strain without the change $(p=0.073)$. In the complementary experiment, we engineered an A to T mutation in low transcription strain 126 and this led to a $20 \%$ increase in the level of cagA mRNA compared to its isogenic control $(p=0.002)$.

Conclusion Presence of $\mathrm{a} T$ at position -54 within the inverted repeat of the cagA promoter region is an important natural determinant of higher levels of cagA transcription. We speculate that this may help explain why only some cag $A^{+} H$ pylori strains cause disease.

Competing interests None declared.

\section{PWE-160 LIVER RESECTION IN METASTATIC GASTROINTESTINAL STROMAL TUMOURS}

doi:10.1136/gutjnl-2012-302514d.160

S S Mudan, A B Fajardo-Puerta. * Department of General surgery, The Royal Marsden, London, UK

Introduction The benefit, if any and case selection for operation in metastatic GIST has not as yet been evaluated. We report our experience with patients undergoing liver resection for metastatic GIST.

Methods From a prospectively held data base spanning 2000-2011 we identified 12 patients who underwent liver resection and cases notes reviewed. Non-parametric statistics were applied.

Results The M:F ratio was 5:7. The median age at diagnosis of the primary tumour was $55 \mathrm{yr}$, range $47-71 \mathrm{yr}$. The site of primary was: Gastric 6, duodenum 1, small intestine 2, colon 2, rectum 1. In three cases liver metastases were present at diagnosis of the primary and these patients underwent synchronous resection of the primary and liver. In the remainder the median disease free interval was $12 \mathrm{~m}$ $(2-96 \mathrm{~m})$. In nine cases the pattern of metastatic disease was hepatic alone, two cases had in addition peritoneal disease and both had had percutaneous biopsy of the primary tumour. One case had local recurrence. All but one patient received neoadjuvant chemotherapy with imatinib and in two cases 2 nd-line treatment with sunitinib and 13 rd-line with nilotinib. The median duration of systemic therapy before operation was $18 \mathrm{~m}$ (10-84) and systemic therapy was stopped after plateau of response or evidence of progression, non-responders were not considered for resection. Liver resections performed: Right extended 1, right 3, left 1, non-anatomic or segmental 7. Additional visceral resection required in 2 (synchronous primary cases excepted). At a median follow-up time of $43 \mathrm{~m}$ from liver resection the status is: NED 7, AWD 3, DOC 1 DOD 1. There was no 30-day mortality.

Conclusion The safety of hepatectomy for GIST in the imatinib treated patient is demonstrated. Whether resection of metastatic disease translates into cure, at least in some patients is yet to be proven but it is suggested that the indications for liver resection for metastatic disease might be extended to this disease.

Competing interests None declared.

\section{PWE-161 PLATELET ACTIVATION IN ACUTE UPPER GASTROINTESTINAL BLEEDING}

doi:10.1136/gutjnl-2012-302514d.161

${ }^{1} \mathrm{~B}$ R Disney, ${ }^{*} \mathrm{R}$ Watson, ${ }^{2} \mathrm{~A}$ Blann, ${ }^{2} \mathrm{G}$ Lip, ${ }^{3} \mathrm{C}$ Tselepis, ${ }^{1} \mathrm{M}$ Anderson. ${ }^{1}$ Department of Gastroenterology, Sandwell and West Birmingham Hospitals NHS Trust, Birmingham, UK; ${ }^{2}$ Department of Cardiology, Sandwell and West Birmingham Hospitals NHS Trust, Birmingham, UK; ${ }^{3}$ Department of Cancer Sciences, University of Birmingham, Birmingham, UK

Introduction Acute upper gastrointestinal bleeding (AUGIB) is a common reason for medical admissions and is associated with significant morbidity and mortality. Studies have previously noted an excess of cardiovascular events in patients who have suffered from AUGIB. Patients who have aspirin withheld for 8 weeks following admission with AUGIB have significantly higher rates of CVS events. The aim of the study was to assess the level of platelet activation, and platelet reactivity, in patients presenting with AUGIB

Methods Patients admitted to Sandwell and West Birmingham Hospitals NHS Trust with AUGIB were recruited. Dyspeptic patients attending for diagnostic OGD were used as controls. To assess platelet activation citrated whole blood was incubated at room temperature with monoclonal mouse antibodies against constitutively expressed platelet marker CD42a-PerCP, and markers of platelet activation PAC1-FITC, and CD62P-APC. Negative controls were run in parallel. Incubation was terminated after $15 \mathrm{~min}$. Platelet reactivity to an agonist, in this case ADP, was assessed by stimulating blood with $\mathrm{ADP}$ for 2 min prior to incubation with antibodies as described above. Samples were analysed using a FACSCalibur flow cytometer. Platelets were identified on the basis of their forward and side scatter properties and the presence of the CD42a platelet-specific marker. CD62P and PAC1 expression were measured by the percentage of platelets expressing these markers. Statistical significance of mean platelet activation was determined by the t-test. The Mann-Whitney $U$ test was utilised for non-normally distributed data. Statistical analysis was performed using SPSS V.18.0 software.

Results A total of 31 patients with AUGIB and 25 controls were recruited. The groups were age and gender matched. The mean age of the AUGIB group is $66.4 \pm 18.2$ years, and the control group $62.8 \pm 6.1$ years. There was a significant differences in the level of CD62P positivity between the study groups $(18.4 \pm 5.8 \%$ in AUGIB group and $13.9 \pm 3.7 \%$ in the control group, $p=0.001)$ and in those staining positive for both CD62P and PAC1 $(1.9 \pm 1.45 \%$ in AUGIB group and $1.2 \pm 1.0 \%$ in the control group, $p=0.027)$. No differences were seen in PAC1 positivity between the groups $(7.1 \pm 5.2$ vs $5.1 \pm 4.2, p=0.127)$. No differences were seen in the response of platelets to ADP between the study and control groups.

Conclusion Patients presenting with AUGIB have higher levels of platelet activation when compared to controls. Platelet reactivity to 